\title{
A Kind of Block Inverse Jacket Transform
}

\section{Dazu Huang ${ }^{a}$, Dan Song, Jianquan Xie and Rongliang Xiao}

\author{
Department of Information Management, Hunan University of Finance and Economics, Changsha \\ 410205, China. \\ ahdzwysj@126.com
}

Keywords: Cocyclic block inverse transform, Orthogonal transforms, Jacket Matrix

\begin{abstract}
A novel block inverse Jacket transform is proposed. A cocyclic block inverse Jacket matrix is constructed in which the high-order cocyclic block inverse Jacket matrix can be decomposed into the low-order sparse cocyclic block inverse Jacket matrices with a successive block architecture, instead of the conventional block inverse Jacket matrix(BIJM). It is a fast algorithm by using recursive mode that leads to reducing computational load.
\end{abstract}

\section{Introduction}

The interesting orthogonal matrices, such as the element-wise or block-wise inverse Jacket matrices, have been developed with orthogonal transforms widely employed in images processing, coding, and other areas [1-3]. More details of these matrices can be referred to [4-8].

Definition I : A $n \times n$ matrix $J_{n}=\left(\alpha_{i j}\right)_{n \times n}$ is called the element-wise inverse Jacket matrix (EIJM) of order $n$ if its inverse matrix $J_{n}^{-1}$ can be obtained by its element-wise inverse.

Many interesting orthogonal matrices belong to the Jacket matrix. With the rapid technological development, different forms of such transforms were improved and generalized. It has been discovered that the newly proposed transforms have been widely used in various signal processing, CDMA, cooperative relay MIMO system [9-13].

Recently, the BIJM $[J]_{n}$ has been investigated while the complex unit $\exp ^{\sqrt{-1}(2 \pi / p)}$ of the EIJM $J_{n}$ is substituted for a suitable matrix unit [7-8]. However, the CBIJM does not attract much attention even though the cocyclic matrix has been very useful for the data coding and processing [14-15].

Definition II : If $\varsigma$ is a finite group of order $\gamma$ with operation $\circ$ and $C$ is a finite Abelian group of order $t$, a co-cycle is a mapping $\phi: \varsigma \times \varsigma \rightarrow C$ satisfying $\phi(a, b) \phi(a \circ b, c)=\phi(a, b \circ c) \phi(b, c)$ where $a, b, c \in \varsigma$ A square matrix $M(\phi)$ whose row $a$ and column $b$ can be indexed by $\varsigma$ with entry $\phi(a, b) \in \varsigma$ in position $(a, b)$ under some fixed ordering, i.e., $M(\phi)=(\phi(a, b))_{(a, b) \in \varsigma}$, is called a cocyclic matrix. If $\phi(1,1)=1$, then it is the normalized cocyclic matrix [14-15].

DefinitionIII: Let $J_{p}=\left(\omega^{\langle i \circ j\rangle_{p}}\right)_{p \times p}, \forall i, j \in \mathrm{Z}_{n}:=\{0,1, \cdots, \mathrm{p}-1\}$, be a matrix of order $\mathrm{p}$, where $\omega=\exp (\sqrt{-1} 2 \pi / p)$ and $\langle i \circ j\rangle_{p}=i \times j \bmod p$, i.e., the subscript $p$ implies modulo $-p$ arithmetic for the argument. Then the matrix $J_{p}$ and its $s$-fold matrix of order $p^{s} J_{p^{s}}=J_{p}^{\otimes s}=\underbrace{J_{p} \otimes J_{p} \cdots \otimes J_{p}}_{s}$ are the conventional cocyclic element inverse Jacket matrices (CEIJM), where $\otimes$ denotes the Kronecker product and $p$ is a prime number.

As a generation of the Hadamard matrix, BIJM inherits the merits of the Hadamard matrix. The inverse transform can be easily obtained by the reciprocal relationships and the fast algorithms. However, versions of cocyclic block-wise inverse Jacket matrix (CBIJM) are still absent [10]. The purpose of this paper is to develop the CBIJM and its generalizations, instead of the CEIJM.

This paper is organized as follows. Section II presents a framework of the fast CBIJT. Section III reports the p-order CBIJM. Section IV discusses the multi-fold CBIJM. Finally, conclusions are drawn in Section V. 


\section{Cocyclic Block Inverse Transforms}

Based on the one-dimensional BIJM $[J]_{p}$, which can be partitioned to the $p \times p$ block matrix, we can transform a suitable vector $x$ into another vector $y$ through a BIJT, i.e., $y=[J]_{p} x$.

In order to derive the CBIJT, we denote a matrix unit by $\alpha$ such that $\alpha^{p}=I_{p}$ for a given prime number $p$, where $I_{p}$ denotes the $p \times p$ identity matrix. As an example, let $\alpha$ be a square matrix of size $2 \times 2$ defined as

$$
\alpha=\left(\begin{array}{ll}
0 & 1 \\
1 & 0
\end{array}\right)
$$

It is easy to prove that $\alpha^{2}=I_{2}$. Actually, matrix $\alpha$ has been employed for the existence of the BIJM [3]. Fortunately, it will be shown that the $s$-fold block Jacket matrix $[J]_{2^{s}} \underline{\underline{\Delta}} \alpha^{\otimes_{s}}$ is also a CBIJM.

We illustrate the cocyclicity of the BIJM $[J]_{p^{s}}$ based on the matrix unit $\alpha$ of size $p \times p$. In particular for the given prime number $p$ we define the matrix unit $\alpha^{h}=\left[e_{i, j}\right]_{p}$, where

$$
e_{i, j}=\left\{\begin{array}{l}
1, \text { for } i=\langle j+h\rangle_{p} ; \\
0, \text { otherwise }
\end{array}\right.
$$

where $\langle j+h\rangle_{p}=j+h \bmod p, \forall i, j, h \in Z_{p}:=\{0,1, \cdots, p-1\} . A:=\left\{\alpha^{h}: h \in Z_{p}\right\}$ forms an Abelian group with the matrix multiplication.

Example I : Let $p=3$, and we have

$$
I_{3}=\alpha^{0}=\left(\begin{array}{lll}
1 & 0 & 0 \\
0 & 1 & 0 \\
0 & 0 & 1
\end{array}\right), \alpha^{1}=\left(\begin{array}{lll}
0 & 0 & 1 \\
1 & 0 & 0 \\
0 & 1 & 0
\end{array}\right), \alpha^{2}=\left(\begin{array}{lll}
0 & 1 & 0 \\
0 & 0 & 1 \\
1 & 0 & 0
\end{array}\right) .
$$

Obviously $Z_{p}$ with the multiplication operation $\langle a \cdot b\rangle_{p}$ is a finite field of order $p$. For $\forall a, x \in Z_{p}$, we define an multiplication function $f_{a}(x)$ over $Z_{p}$, i.e., $f_{a}(x):=\langle a \cdot x\rangle_{p}$. With the aid of the multiplication function $f_{a}(x)$, we define a block matrix of size $p \times p^{2}$ by concatenating $p$ matrices $\alpha^{h_{i}}$ of size $p \times p, \forall h_{i} \in Z_{p}$, i.e., $[\beta]:=\left[\alpha^{h_{0}}, \alpha^{h_{1}}, \cdots \alpha^{h_{p-1}}\right]$, and hence

$$
\left[\beta_{a}\right]:=\left[\alpha^{f_{a}\left(h_{0}\right)}, \alpha^{f_{a}\left(h_{1}\right)}, \cdots \alpha^{f_{a}\left(h_{p-1}\right)}\right] .
$$

Lemma I :For block matrices $\left[\beta_{\alpha}\right]$ and $\left[\beta_{b}\right], \forall a, b \in Z_{p}$, we have $\left[\beta_{a}\right] \cdot\left[\beta_{\mathrm{b}}\right]^{\mathrm{T}}= \begin{cases}p I, & \text { for }\langle a+b\rangle_{p}=0 \\ 0, & \text { for }\langle a+b\rangle_{p} \neq 0 .\end{cases}$

Proof: If $a=b=0$, the $\left[\beta_{0}\right]=[I, I, \cdots, I]$, and hence $\left[\beta_{0}\right] \cdot\left[\beta_{0}\right]^{T}=p I$. If $\langle a+b\rangle_{p}=0, \forall a, b \in \mathrm{Z}_{p}$, then for $\forall h_{i} \in Z_{p}, f_{a}\left(h_{i}\right)+f_{b}\left(h_{i}\right)=\left\langle a h_{i}\right\rangle_{p}+\left\langle b h_{i}\right\rangle_{p}=\left\langle(a+b) h_{i}\right\rangle_{p}=0$. Therefore, it is easy to verify that $\left[\beta_{a}\right] \cdot\left[\beta_{b}\right]^{T}=\sum_{i=1}^{p} \alpha^{f_{a}\left(h_{i}\right)+f_{b}\left(h_{i}\right)}=p I$. But if $\langle a+b\rangle_{p} \neq 0$, for $0<\langle a+b\rangle_{p}<p,\left\{\langle c(a+b)\rangle_{p}: c \in \mathrm{Z}_{p}\right\}=\mathrm{Z}_{p}$. Consequently, we have $\left[\beta_{a}\right] \cdot\left[\beta_{b}\right]^{T}=\sum_{i=0}^{p-1} \alpha^{i}$, which can be proved to be equal to zero since $\alpha^{p}-I=0$ but for $\alpha \neq I$.

Example II : Let us consider $\alpha$ with $p=2$ in (1). It is obvious that $\alpha^{2}=I$ is an identity matrix of $\operatorname{size} 2 \times 2 . \operatorname{Let}[\beta]=\left[\alpha^{0}, \alpha^{1}\right]$, then $\left[\beta_{0}\right]=\left[\alpha^{0}, \alpha^{0}\right]=\left[\begin{array}{llll}1 & 0 & 1 & 0 \\ 0 & 1 & 0 & 1\end{array}\right],\left[\beta_{1}\right]=\left[\alpha^{0}, \alpha^{1}\right]=\left[\begin{array}{cccc}1 & 0 & 0 & 1 \\ 0 & 1 & 1 & 0\end{array}\right]$ 
It is straightforward to show that

$\left[\beta_{0}\right] \cdot\left[\beta_{0}\right]^{T}=\left[\beta_{1}\right] \cdot\left[\beta_{1}\right]^{T}=2 I_{2}$.

\section{The p-order CBIJM}

In [7-8], Lee et al . expanded the EIJM to BIJM.

Definition IV: A $n p \times n p$ block matrix $[J]_{n}=\left(\left[\alpha_{i j}\right]_{p}\right)_{n p \times n p}$ is called the BIJM of order $n$ if $[J]_{n}^{-1}=$ $=\frac{1}{c}\left(\left[\alpha_{i j}\right]^{-1}\right)_{n p \times n p}^{T}$, where $c$ is the normalized value and $\left[\alpha_{i j}\right]_{p \times p}$ denotes a matrix unit of size $p \times p$.

Definition V: For a given prime number $p$, let $\alpha$ be a $p \times p$ matrix unit such that $\alpha_{p}=I$ and $[\beta]=\left[\alpha^{0}, \alpha^{1}, \cdots, \alpha^{p-1}\right]$. Define the $p$-order BIJM $[J]_{p}$ of size $p^{2} \times p^{2}$ as follows

$$
[J]_{P}:=\left[\begin{array}{c}
{\left[\beta_{0}\right]} \\
{\left[\beta_{1}\right]} \\
{\left[\beta_{2}\right]} \\
\vdots \\
{\left[\beta_{p-1}\right]}
\end{array}\right]=\left[\begin{array}{cccc}
\alpha^{0} & \alpha^{0} & \cdots & \alpha^{0} \\
\alpha^{0} & \alpha^{1} & \cdots & \alpha^{p-1} \\
\alpha^{0} & \alpha^{2} & \cdots & \alpha^{2(p-1)} \\
\vdots & \vdots & \ddots & \vdots \\
\alpha^{0} & \alpha^{p-1} & \cdots & \alpha^{(p-1)(p-1)}
\end{array}\right]
$$

and thus its inverse

$$
[J]_{p}^{-1}:=\frac{1}{p}\left[\begin{array}{cccc}
\alpha^{0} & \alpha^{0} & \cdots & \alpha^{0} \\
\alpha^{0} & \alpha^{\langle-1\rangle_{p}} & \cdots & \alpha^{\langle-(p-1)\rangle_{p}} \\
\alpha^{0} & \alpha^{\langle-2\rangle_{p}} & \cdots & \alpha^{\langle-2(p-1)\rangle_{p}} \\
\vdots & \vdots & \ddots & \vdots \\
\alpha^{0} & \alpha^{\langle-(p-1)\rangle_{p}} & \cdots & \alpha^{\langle-(p-1)(p-1)\rangle_{p}}
\end{array}\right]
$$

Consequently, we have $[J]_{p} \cdot[J]_{p}^{-1}=[J]_{p}^{-1} \cdot[J]_{p}=I_{p^{2} \times p^{2}}$.

Example III: Taking $\left[\beta_{0}\right]$ and $\left[\beta_{1}\right]$ for $p=2$, we have

$$
[J]_{2}=\left[\begin{array}{cc}
\alpha^{0} & \alpha^{0} \\
\alpha^{0} & \alpha^{1}
\end{array}\right]=\left[\begin{array}{cccc}
1 & 0 & 1 & 0 \\
0 & 1 & 0 & 1 \\
1 & 0 & 0 & 1 \\
0 & 1 & 1 & 0
\end{array}\right],
$$

and its inverse

$$
[J]_{2}^{-1}=\frac{1}{2}\left[\begin{array}{cc}
\alpha^{0} & \alpha^{0} \\
\alpha^{0} & \alpha^{\langle-1\rangle_{2}}
\end{array}\right]=\frac{1}{2}\left[\begin{array}{cccc}
1 & 0 & 1 & 0 \\
0 & 1 & 0 & 1 \\
1 & 0 & 0 & 1 \\
0 & 1 & 1 & 0
\end{array}\right]
$$

Actually, we have

$$
[J]_{2}[J]_{2}^{-1}=\left[\begin{array}{cc}
\alpha^{0} & \alpha^{0} \\
\alpha^{0} & \alpha^{1}
\end{array}\right]\left[\begin{array}{cc}
\alpha^{0} & \alpha^{0} \\
\alpha^{0} & \alpha^{1}
\end{array}\right]=\left[\begin{array}{cc}
I_{2} & 0 \\
0 & I_{2}
\end{array}\right],
$$

where $\alpha^{0}+\alpha^{1}=0$ since $\alpha^{2}=I$ and $\alpha \neq I$ over the finite field.

We note that the above-mentioned BIJM was first proposed by Lee and Hou [7] for the proof of existence of Jacket matrices over the finite field. Next, we illustrate that this BIJM is also a CBIJM in essence. 
Theorem I : Let $\varsigma=\mathrm{Z}_{P}$ with an operation $a \circ b:=\langle a+b\rangle_{p}, \forall \mathrm{a}, b \in \mathrm{Z}_{\mathrm{p}}$, and $C:=\left\{\alpha^{i}: i \in \mathrm{Z}_{p}\right\}$ with the traditional multiplication. The BIJM $[J]_{p}$ in (6) whose rows and columns are both indexed in ${ }_{\varsigma}$ under the increasing order (i.e., $0 \prec 1 \prec \cdots \prec p-1)$ and entries $\phi(a, b)$ in position $(a, b)$ is the CBIJM.

Proof: According to the defined BIJM $[J]_{p}$ in $(6)$, we have $\phi(a, b):=\alpha^{\langle a \cdot b\rangle_{p}}$. For $\forall c \in \mathrm{Z}_{p}$, we have

$$
\phi(a, b) \phi(a \circ b, c)=\alpha^{\langle a \cdot b\rangle_{p}} \cdot \alpha^{\langle(a+b) \cdot c\rangle_{p}}=\alpha^{\langle a \cdot b+(a+c) \cdot c\rangle_{p}} .
$$

On the other hand,

$$
\phi(a, b \circ c) \phi(b, c)=\alpha^{\langle a \cdot(b+c)\rangle_{p}} \cdot \alpha^{\langle b \cdot c\rangle_{p}}=\alpha^{\langle a \cdot(b+c)+b \cdot c\rangle_{p}} .
$$

Combining (11) and (12), we have

$$
\phi(a, b) \phi(a \circ b, c)=\phi(a, b \circ c) \phi(b, c) .
$$

Thus the BIJM $[J]_{p}$ is also a CBIJM.

\section{The Multi-fold CBIJM}

$\mathrm{n}$ order to derive the high-order recursive CBIJM $[J]_{p^{s}}$ for any prime number $p$ and nonnegative integer $s$, let us introduce some lemmas [1-2].

Lemma II : Let $A, B, C, D$ are matrices with suitable sizes. We have $(A \otimes B) \cdot(C \otimes D)=(A \cdot C) \otimes(B \cdot D),(A \otimes B)^{-1}=\left(A^{-1} \otimes B^{-1}\right),(A \otimes B)^{T}=\left(A^{T} \otimes B^{T}\right)$.

Theorem 2: For a given prime number $p$, let $[A]_{p}=\left[\alpha_{i, j}\right]_{p}$ and $[B]_{p}=\left[\gamma_{s, t}\right]_{p}, \forall i, j, s, t \in \mathrm{Z}_{p}$, be two CBIJMs of order $p$ that correspond to the matrix units $\alpha$ and $\gamma$ such that $\alpha^{p}=I$ and $\gamma^{p}=I$, respectively. Then the 2 -fold Kronecker product matrix $[J]_{p^{2}}=[A]_{p} \otimes[B]_{p}$ is a 2-fold CBIJM of order $p^{2}$.

Proof: Since $[A]_{p}=\left[\alpha_{i, j}\right]_{p}$ and $[B]_{p}=\left[\gamma_{s, t}\right]_{p}$ are both BIJM, we have the inverse

$$
[A]_{p}^{-1}=\frac{1}{p}\left[\alpha_{i, j}^{-1}\right]_{p}^{T}, \quad[B]_{p}^{-1}=\frac{1}{p}\left[\gamma_{s, t}^{-1}\right]_{p}^{T} .
$$

Let $[A]_{p} \otimes[B]_{p}=\left[\sigma_{i p+s, j p+t}\right]_{p^{2}}$, where $\sigma_{i p+s, j p+t}=\alpha_{i, j} \cdot \gamma_{s, t}$ denotes the traditional multiplication of two matrices. Therefore, we have the inverse matrix $[J]_{p^{2}}^{-1}$ that can be calculated directly from the block inverse of the original block matrix $[J]_{p^{2}}$, i.e.,

$$
[J]_{p^{2}}^{-1}=\left([A]_{p} \otimes[B]_{p}\right)^{-1}=\left([A]_{p}^{-1} \otimes[B]_{p}^{-1}\right)=\frac{1}{p^{2}}\left[\alpha_{i, j}^{-1} \cdot \gamma_{s, t}^{-1}\right]_{p^{2}}^{T}=\frac{1}{p^{2}}\left[\sigma_{i p+s, j p+t}^{-1}\right]_{p^{2}}^{T} .
$$

It implies that $[J]_{p^{2}}$ is a block Jacket matrix.

Next, we show that matrix $[J]_{p^{2}}$ is a CBIJM under the indexed row and column. Assume that $[A]_{p}$ and $[B]_{p}$ are both CBIJMs under the row and column index over $Z_{p}$, respectively

$$
\left\{\begin{array}{l}
a_{s 1} \prec a_{s 2} \prec \cdots a_{s^{p}}, \quad \text { for } a_{s j} \in \mathrm{Z}_{p}, \forall j \in \mathrm{Z}_{p} \\
b_{s 1} \prec b_{s 2} \prec \cdots b_{s^{p}}, \quad \text { for } b_{s k} \in \mathrm{Z}_{p}, \forall k \in \mathrm{Z}_{p}
\end{array} ;\right.
$$

where $s \in\{r, c\}, a_{r j}$ and $a_{c j}$ denote the $j^{\text {th }}$ row and the $j^{\text {th }}$ column index of block matrix $[A]_{p}, b_{r k}$ and $b_{c k}$ denote the $k^{\text {th }}$ row and the $k^{\text {th }}$ column index of block matrix $[B]_{p}$, and $\prec$ denotes the increasing order. Then for the $p^{2}$-order block matrix $[J]_{p^{2}}$ over $Z_{p^{2}}$, the row and column index order can be defined as follows 


$$
a_{s j} b_{s k} \prec a_{s i} b_{s h}, \text { if }\left\{\begin{array}{c}
a_{s j} \prec a_{s i} ; \\
a_{s j}=a_{s i}, b_{s k}
\end{array} b_{s h .}\right.
$$

Also the entries of $[J]_{p^{2}}$ are defined on the basis of $[J]_{p}$ as $\phi_{p^{2}}\left(a_{r i} b_{r h}, a_{c j} b_{c k}\right)=\phi_{p}\left(a_{r i}, a_{c j}\right) \cdot \phi_{p}\left(b_{r h}, b_{c k}\right)$. As for the entries $\phi_{p}\left(a_{i}, a_{j}\right)$ and $\phi_{p}\left(b_{h}, b_{k}\right)$ of $[A]_{p}$ and $[B]_{p}, \forall a_{i}, a_{j}, a_{l} \in \mathrm{Z}_{p}$ and $\forall b_{h}, b_{k}, b_{t} \in \mathrm{Z}_{p}$, we have

$$
\begin{aligned}
& \phi_{p}\left(a_{i}, a_{j}\right) \phi_{p}\left(a_{i} \circ a_{j}, a_{l}\right)=\phi_{p}\left(a_{i}, a_{j} \circ a_{l}\right) \phi_{p}\left(a_{j}, a_{l}\right), \\
& \phi_{p}\left(b_{h}, b_{k}\right) \phi_{p}\left(b_{h} \circ b_{k}, b_{t}\right)=\phi_{p}\left(b_{h}, b_{k} \circ b_{t}\right) \phi_{p}\left(b_{k}, b_{t}\right),
\end{aligned}
$$

Therefore, it can be easily verified that

$$
\phi_{p^{2}}\left(a_{i} b_{h}, a_{j} b_{k}\right) \phi_{p^{2}}\left(a_{i} b_{h}, a_{j} b_{k} \circ a_{l} b_{t}\right)=\phi_{p^{2}}\left(a_{i} b_{h}, a_{j} b_{k} \circ a_{l} b_{t}\right) \phi_{p^{2}}\left(a_{j} b_{k}, a_{l} b_{t}\right) .
$$

It shows that block matrix $[J]_{p^{2}}$ is also a CBIJM under the indexed order in (17). This completes the proof of this theorem.

Corollary I : For any prime number $p$ and non-negative integer number $s, \operatorname{let}[J]_{p^{s}}=[J]_{p}^{\otimes_{s}}$ be an $s$-fold block matrix, i.e., $[J]_{p^{s}}=\underbrace{[J]_{p} \otimes \cdots[J]_{p}}_{s}$. Then the block matrix $[J]_{p^{s}}$ is a CBIJM of order $p^{s}$.

Consequently, the s-fold CBIJM $[J]_{p^{s}}$ of order $p^{s}$ can be generated from the following factorization algorithm

$$
[J]_{p^{s}}=[J]_{p^{s-1}} \otimes[J]_{p}=\prod_{i=1}^{s}\left(I_{p^{s-i}} \otimes[J]_{p} \otimes I_{p^{i-1}}\right)
$$

where $I_{p^{i}}$ denotes the identity matrix of size $p^{i} \times p^{i}$ and $I_{p^{0}}=1$ for the simple description.

Corollary II: Based on the p-order CBIJM $[J]_{p}$ for any number $p$, the s-fold CBIJM $[J]_{p^{s}}$ of order $p^{s}$ can be constructed with the recursive formula

$$
[J]_{p^{s}}=\prod_{i=1}^{s}\left(I_{p^{s-i}} \otimes[J]_{p} \otimes I_{p^{i-1}}\right),
$$

where $p$ is any prime number and $s$ is a nonnegative integer number.

Proof: We deploy induction on index $s$.If $s=1$, then it is clearly true, i.e., $[J]_{p^{1}}=[J]_{p}$ In what follows, we assume the hypothesis is true for $s$.Namely, for $\forall i \in\{1,2, \cdots, s\}$ we have

$$
[J]_{p^{s}}=\prod_{i=1}^{s}\left(I_{p^{s-i}} \otimes[J]_{p} \otimes I_{p^{i-1}}\right) .
$$

Then we show it must therefore hold for $s+1$. Actually,by induction based on properties of the Kronecker product we have

$$
[J]_{p^{s+1}}=[J]_{p} \otimes[J]_{p^{s}}=\left([J]_{p} \cdot I_{p}\right) \otimes\left(I_{p^{s}} \cdot[J]_{p^{s}}\right)=\left([J]_{p} \otimes I_{p^{s}}\right)\left(I_{p} \otimes[J]_{p^{s}}\right)=\prod_{i=1}^{s+1}\left(I_{p^{s-i}} \otimes[J]_{p} \otimes I_{p^{i-1}}\right)
$$

In order to show the factorization of the generalized CBIJM $[J]_{n}$ of order $p^{s}$ with any prime number $p$, we propose several construction approaches in Table 1. In this table, the second column is the decomposition for the numbers (order) of the CBIJM, and the third column is the construction for CBIJM. It shows that the large-order CBIJM can be designed on the basis of the lower order $\mathrm{CBIJ}[J]_{p}$ with sparse matrices in the successive architecture. 


\section{Conclusion}

A simple method of developing the CBIJM is proposed. This method is presented for its simplicity and clarity, which decomposes the high-order CBIJM into multiple sparse matrices with the lowerorder CBIJMs, instead of the conventional BIJMs or EIJMs.

Table 1 Decomposition of Order for the CBJM $[J]_{p^{s}}$

\begin{tabular}{c|l|c}
\hline Order & Decomposition & \multicolumn{1}{c}{ CBIJM } \\
\hline 2 & $2=2$ & {$[J]_{2}=[J]_{2}$} \\
\hline 3 & $3=3$ & {$[J]_{3}=[J]_{3}$} \\
\hline 4 & $2^{2}=2 \times 2$ & {$[J]_{4}=[J]_{2}^{\otimes 2}$} \\
\hline 5 & $5=5$ & {$[J]_{5}=[J]_{5}$} \\
\hline 7 & $7=7$ & {$[J]_{7}=[J]_{7}$} \\
\hline 8 & $2^{3}=2^{2} \times 2$ & {$[J]_{8}=[J]_{2}^{\otimes 3}$} \\
\hline 9 & $3^{2}=3 \times 3$ & {$[J]_{9}=[J]_{3}^{\otimes 2}$} \\
\hline
\end{tabular}

\section{Acknowledgement}

This work was supported by Research Plan Projects of Science-Technology Department of Hunan Province, also was supported in part by the construct program of the key discipline in Hunan province.

\section{References}

[1] R. K. Yarlagadda and J. E. Hershey, Hadamard Matrix Analysis and Synthesis With Applications to Communications Signal/Image Processing, Kluwer Academic publishers, 1997.

[2] K. J. Horadam, Hadamard Mastrices and Their Applications, Princeton University Press, 2006.

[3] A.T. Butson, Generalized Hadamard matrices, Proc. Amer. Math. Soc., 13(1962) 894-898.

[4] M. H. Lee, The Center Weighted Hadamard Transform, IEEE Trans. Circuits Syst, CAS36(1989) 1247-1252.

[5] M. H. Lee and Y. Guo, A Novel Construction of Jacket Matrix from Characters on finite Abelian Group, Electronics Letters, 46(2010) 39-47.

[6] Z. Chen and M. H. Lee, Fast cocyclic Jacket transform, IEEE Trans. Signal Processing, 56(2008) 2143-2148.

[7] M. H. Lee and J. Hou, Fast block inverse Jacket transform, IEEE Signal Process. Lett., 3(2006) 461-464.

[8] G. Zeng and M. H. Lee, A generalized reverse block Jacket transform, IEEE Trans. Circuits and Systems, 55(2008) 1589-1599.

[9] B. Yuri, S. M. Dodunekov and M. H. Lee, On Odd Order Jacket Matrices Over Finite Character Fields, 12th Algebraic and Combinatorial Coding Theory, Novosibirsk, Russia, Sept. 5-11, 2010.

[10]Z. Chen, M. H. Lee and W. Song, Fast Cocyclic Jacket Transform Based on DFT, IEEE Inter. Conference on Communication, 2008, pp. 766-769,.

[11]M. H. Lee and Y. L. Borissov, A proof of non-existence of bordered jacket matrices of odd order over some fields, Electronics Letters, 46(2010) 349-351. 
[12]W. Song, M. H. Lee and G. Zeng, Orthogonal Space-Time Block Codes Design using Jacket Transform for MIMO Transmission System, IEEE Inter. Conference on Communication, 2008, pp. 766- 769.

[13]X. Jiang, M. H. Lee, Y. Guo, Y. Yan and S. A. Latif, Ternary Codes from Modified Jacket Matrices, J. Communications and Networks, 13(2011).12-16.

[14]K. J. Horadam and P. Udaya,Cocyclic Hadamard Codes,IEEE Trans. Infor. Theory, 46(2000) 1545-1550.

[15]A. A. I. Perera and K. J. Horadam,Cocyclic Generalised Hadamard Matrices and Central RelativeDifference Sets, J. Designs, Codes and Crypt., 15(1998) 187-200. 